\title{
FERMION-BOSON LOOPS WITH BILINEAR $R$-PARITY VIOLATION LEADING TO MAJORANA NEUTRINO MASS AND MAGNETIC MOMENTS
}

\author{
MAREK GÓŹDŹ and WIESŁAW A. KAMIŃSKI \\ Department of Informatics, Maria Curie-Sktodowska University, \\ pl. Marii Curie-Sktodowskiej 5, 20-031 Lublin, Poland \\ mgozdz@kft.umcs.lublin.pl,kaminski@neuron.umcs.lublin.pl
}

\begin{abstract}
We present analytic expressions corresponding to a set of one loop Feynman diagrams, built within $R$-parity violating ( $\mathrm{RpV}$ ) minimal supersymmetric standard model (MSSM). Diagrams involve both bilinear and trilinear $\mathrm{RpV}$ couplings and represent Majorana neutrino masses and magnetic moments.
\end{abstract}

\section{Supersymmetric model with $R$-parity violation}

Building the minimal supersymmetric (SUSY) version of the Standard Model (MSSM) 1 one usually assumes the conservation of the $R$-parity, defined as $R=$ $(-1)^{3 B+L+2 S}$. In theories preserving the $R$-parity the lepton and baryon numbers are conserved, and SUSY particles are not allowed to decay to non-SUSY ones. It implies that the lightest SUSY particle must remain stable, giving a good natural dark matter candidate. The $R$-parity conservation can be achieved by neglecting certain theoretically allowed terms in the superpotential. By retaining these terms one finishes with an $R$-parity violating $(\mathrm{RpV})$ model, $\stackrel{2134}{2}$ with richer phenomenology and many exotic interactions. The $\mathrm{RpV}$ models provide mechanisms of generating Majorana neutrino masses and magnetic moments, describe neutrino decay, SUSY particles decays, exotic nuclear processes like the neutrinoless double beta decay, and many more. Being theoretically allowed, RpV SUSY theories are interesting tools for studying the physics beyond the Standard Model.

The $R$-parity conserving part of the superpotential of MSSM is usually written as

$$
\begin{aligned}
W^{M S S M} & =\epsilon_{a b}\left[\left(\mathbf{Y}_{E}\right)_{i j} L_{i}^{a} H_{1}^{b} \bar{E}_{j}+\left(\mathbf{Y}_{D}\right)_{i j} Q_{i x}^{a} H_{1}^{b} \bar{D}_{j}^{x}\right. \\
& \left.+\left(\mathbf{Y}_{U}\right)_{i j} Q_{i x}^{a} H_{2}^{b} \bar{U}_{j}^{x}+\mu H_{1}^{a} H_{2}^{b}\right],
\end{aligned}
$$

while its $\mathrm{RpV}$ part reads

$$
\begin{aligned}
W^{R p V} & =\epsilon_{a b}\left[\frac{1}{2} \lambda_{i j k} L_{i}^{a} L_{j}^{b} \bar{E}_{k}+\lambda_{i j k}^{\prime} L_{i}^{a} Q_{j x}^{b} \bar{D}_{k}^{x}\right] \\
& +\frac{1}{2} \epsilon_{x y z} \lambda_{i j k}^{\prime \prime} \bar{U}_{i}^{x} \bar{D}_{j}^{y} \bar{D}_{k}^{z}+\epsilon_{a b} \kappa^{i} L_{i}^{a} H_{2}^{b} .
\end{aligned}
$$


The Y's are $3 \times 3$ Yukawa matrices. $L$ and $Q$ are the $S U(2)$ left-handed doublets while $\bar{E}, \bar{U}$ and $\bar{D}$ denote the right-handed lepton, up-quark and down-quark $S U(2)$ singlets, respectively. $H_{1}$ and $H_{2}$ mean two Higgs doublets. We have introduced color indices $x, y, z=1,2,3$, generation indices $i, j, k=1,2,3$ and the $\mathrm{SU}(2)$ spinor indices $a, b=1,2$. One of the major problems in these models is the assurance of proton stability, therefore the baryon number violating coupling constant $\lambda^{\prime \prime}$ is usually set to zero. It is also customary to neglect the bilinear $\left(\kappa^{i}\right)$ term and concentrate on the phenomenology of the remaining trilinear terms.

In the present paper we introduce processes which involve both bi- and trilinear coupling constants. We focus on one loop Feynman diagrams which potentially lead to Majorana neutrino masses and magnetic moments. Such diagrams have been proposed by J. D. Vergados 5$]$ and, to our best knowledge, this is the first attempt to treat them in an exact analytical way. In the next section we present the full set of new Feynman diagrams within the RpV MSSM model, together with the corresponding analytical functions. We do not have any numerical results yet. The work on a stable renormalization group based code, which would include the $\mathrm{RpV}$ effects, is in progress.

One of the characteristic features of the MSSM is that the lepton doublet superfield $L$ has the same quantum numbers as the $H_{1}$ Higgs doublet superfield. Thus in the $\mathrm{RpV}$ models one can combine them into one superfield $\mathcal{L}_{\alpha}^{a}=\left\{H_{1}^{a}, L_{1,2,3}^{a}\right\}, \alpha=$ $0, \ldots, 3$. This observation gives rise to the bilinear $\kappa \mathrm{RpV}$ term. Summarizing, in full $\mathrm{RpV}$ one has to take into account not only the usual trilinear vertices but also the bilinear ones, which represent the mixing between SUSY superfields. In the case of one loop diagrams the bilinear insertions may be placed on the external neutrino lines (neutrino-neutralino mixing) as well as inside the loop on the internal fermion or boson line, or both. In this communication we concentrate on the bilinear couplings inside the loop only, leaving the full discussion to a forthcoming regular paper.

\section{General diagrams leading to Majorana neutrino masses}

Except the usually discussed lepton-slepton and quark-squark loops,, 6 one may construct new class of diagrams which contain bilinear $\mathrm{RpV}$ insertions on the internal fermion or boson lines. These diagrams give raise to the Majorana neutrino mass term exactly in the same way as the usual ones.

The neutrino mass term will be proportional to the product of all the coupling constants (two trilinear and two bilinear ones, the latter having proper mass dimension) times the function coming from integration over 4-momentum of all the propagators. To present the results in a compact form we first define two functions, $\mathcal{F}_{1}$ and $\mathcal{F}_{2}$ as follows:

$$
\mathcal{F}_{1}(\alpha, \beta, \gamma, \delta)=\int_{-\infty}^{\infty} \frac{d^{4} k}{(2 \pi)^{4}} \frac{1}{\left(k^{2}-m_{\alpha}^{2}\right)\left(k^{2}-m_{\beta}^{2}\right)\left(k^{2}-m_{\gamma}^{2}\right)\left(k^{2}-m_{\delta}^{2}\right)}
$$




$$
\begin{aligned}
& =\frac{1}{16 \pi^{2}}\left[\frac{m_{\alpha}^{2}}{\left(m_{\alpha}^{2}-m_{\beta}^{2}\right)\left(m_{\alpha}^{2}-m_{\gamma}^{2}\right)\left(m_{\alpha}^{2}-m_{\delta}^{2}\right)} \ln \left(\frac{m_{\alpha}^{2}}{m_{\gamma}^{2}}\right)\right. \\
& +\frac{m_{\beta}^{2}}{\left(m_{\beta}^{2}-m_{\alpha}^{2}\right)\left(m_{\beta}^{2}-m_{\gamma}^{2}\right)\left(m_{\beta}^{2}-m_{\delta}^{2}\right)} \ln \left(\frac{m_{\beta}^{2}}{m_{\gamma}^{2}}\right) \\
& \left.+\frac{m_{\delta}^{2}}{\left(m_{\delta}^{2}-m_{\alpha}^{2}\right)\left(m_{\delta}^{2}-m_{\beta}^{2}\right)\left(m_{\delta}^{2}-m_{\gamma}^{2}\right)} \ln \left(\frac{m_{\delta}^{2}}{m_{\gamma}^{2}}\right)\right] \\
\mathcal{F}_{2}(\alpha, \beta, \gamma, \delta) & =\int_{-\infty}^{\infty} \frac{d^{4} k}{(2 \pi)^{4}} \frac{k^{2}}{\left(k^{2}-m_{\alpha}^{2}\right)\left(k^{2}-m_{\beta}^{2}\right)\left(k^{2}-m_{\gamma}^{2}\right)\left(k^{2}-m_{\delta}^{2}\right)} \\
& =\frac{1}{16 \pi^{2}}\left[\frac{m_{\alpha}^{4}}{\left(m_{\alpha}^{2}-m_{\beta}^{2}\right)\left(m_{\alpha}^{2}-m_{\gamma}^{2}\right)\left(m_{\alpha}^{2}-m_{\delta}^{2}\right)} \ln \left(\frac{m_{\alpha}^{2}}{m_{\gamma}^{2}}\right)\right. \\
& +\frac{m_{\beta}^{4}}{\left(m_{\beta}^{2}-m_{\alpha}^{2}\right)\left(m_{\beta}^{2}-m_{\gamma}^{2}\right)\left(m_{\beta}^{2}-m_{\delta}^{2}\right)} \ln \left(\frac{m_{\beta}^{2}}{m_{\gamma}^{2}}\right) \\
& \left.+\frac{m_{\delta}^{4}}{\left(m_{\delta}^{2}-m_{\alpha}^{2}\right)\left(m_{\delta}^{2}-m_{\beta}^{2}\right)\left(m_{\delta}^{2}-m_{\gamma}^{2}\right)} \ln \left(\frac{m_{\delta}^{2}}{m_{\gamma}^{2}}\right)\right]
\end{aligned}
$$

The indices $\alpha, \beta, \ldots$ label particles with masses $m_{\alpha}, m_{\beta}, \ldots$, respectively, appearing inside the loop. One can check that both of theses functions are symmetric with respect to permutation of any two masses.

We present the three new diagrams together with the corresponding loop functions in Tab. 1 below. We use the convention that fermions inside the loop are successively labeled by $f 1, f 2$, and $f 3$, and similarly the bosons by $b 1, b 2$, and $b 3$. If the loop contains only one particle of given spin, it is labeled simply by $f$ or $b$.

Table 1. Loop integrals governing Majorana neutrino mass.

Integral $\mathcal{I}$




\section{General diagrams leading to Majorana neutrino transition magnetic moments}

The magnetic moment of the Majorana neutrino is proportional to the amplitude of an effective neutrino-photon-neutrino interaction. Therefore an external photon has to be added to the diagrams considered in the previous section. The photon may interact only with particles possessing non-zero electric charge, so it may be attached only to the internal lines of the loop. The external lines contain neutrinos or, more exactly, a neutrino-neutralino superposition, which is electrically neutral.

As before, we start by calculating two integrals in the momentum space, which represent the situation when the photon interacts with the particle with mass $m_{\alpha}$ :

$$
\begin{aligned}
\mathcal{F}_{3}(\alpha, \beta, \gamma, \delta) & =\int_{-\infty}^{\infty} \frac{d^{4} k}{(2 \pi)^{4}} \frac{1}{\left(k^{2}-m_{\alpha}^{2}\right)^{2}\left(k^{2}-m_{\beta}^{2}\right)\left(k^{2}-m_{\gamma}^{2}\right)\left(k^{2}-m_{\delta}^{2}\right)} \\
& =-\frac{1}{2 m_{\alpha}} \frac{\partial}{\partial m_{\alpha}} \mathcal{F}_{1}(\alpha, \beta, \gamma, \delta), \\
\mathcal{F}_{4}(\alpha, \beta, \gamma, \delta) & =\int_{-\infty}^{\infty} \frac{d^{4} k}{(2 \pi)^{4}} \frac{k^{2}}{\left(k^{2}-m_{\alpha}^{2}\right)^{2}\left(k^{2}-m_{\beta}^{2}\right)\left(k^{2}-m_{\gamma}^{2}\right)\left(k^{2}-m_{\delta}^{2}\right)} \\
& =-\frac{1}{2 m_{\alpha}} \frac{\partial}{\partial m_{\alpha}} \mathcal{F}_{2}(\alpha, \beta, \gamma, \delta) .
\end{aligned}
$$

The resulting loop integrals are presented in Tab. 2. For the sake of simplicity, we have not taken into account the possible permutations of particles inside the loop. For example, in the case labeled "IV" the photon could be attached also to the fermion $f 2$, which means that one should add a $+(f 1 \leftrightarrow f 2)$ term to the expression listed in the table. An interesting observation is, that some of the processes ("V" and "VII") are strictly forbidden. The zeros come out as an exact result of $k$ integration of an odd $k$-function.

\section{Summary}

In this paper we have discussed the up-to-date problem of generating Majorana neutrino mass and transition magnetic moments. The well known mechanism, which exists within supersymmetric standard model with non-conserved $R$-parity, has been extended to include also processes described by bilinear terms in the superpotential. We have limited ourselves to the case when bilinear insertions are present on the internal lines of the fermion-boson loop. The remaining possibility, where the insertions are on the external neutrino lines, are possible due to the fact that in $\mathrm{RpV}$ models neutrinos mix with neutralinos. We will discuss this problem in details elsewhere.

The lowest level diagrams of interest have been solved and the analytic formulae can be found in Tab. 1 and 2 Using these functions the resulting mass $\mathcal{M}$ and magnetic moment $\mu$ can be written as follows:

$$
\mathcal{M}=c C_{1} C_{2} X_{1} X_{2} \mathcal{I},
$$


Table 2. Loop integrals governing Majorana neutrino transition magnetic moments.

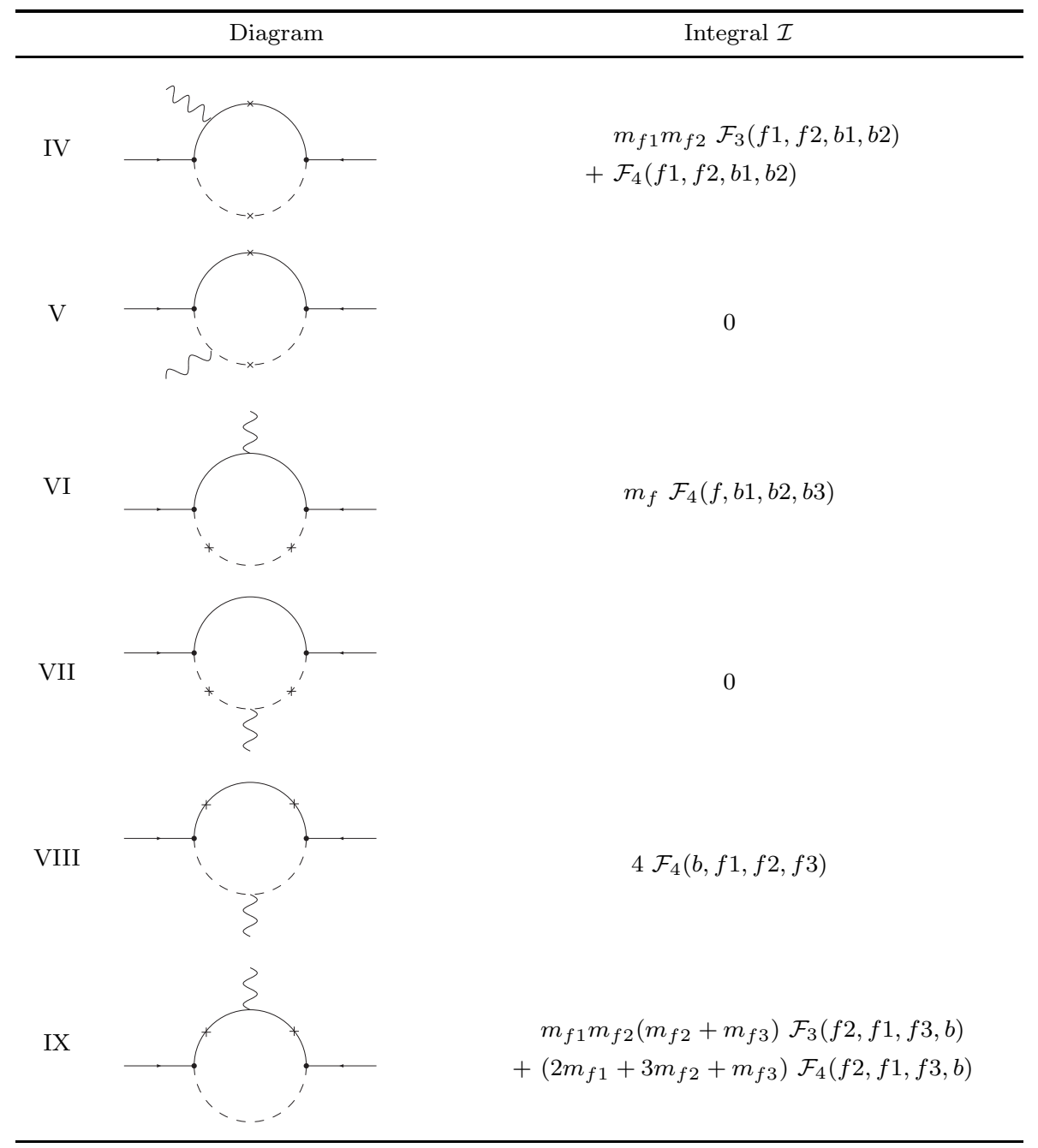

$$
\mu=c C_{1} C_{2} X_{1} X_{2} Q \mathcal{I} 2 m_{e} \mu_{B},
$$

where the dimensionless trilinear coupling constants have been denoted by $C_{1,2}$, the bilinear coupling constants by $X_{1,2}, \mathcal{I}$ is the appropriate integral function, $\mu_{B}$ is the Bohr magneton, and $m_{e}$ the electron mass. $c$ is the color factor and is equal to 3 whenever quarks or squarks appear in the loop; it is equal to 1 in all other cases. $Q$ is a dimensionless number and denotes the electric charge of the particle to which the photon is attached, in units of the elementary charge $e$.

It is not difficult to check that bilinear couplings appearing on fermion lines have exactly the dimension of mass $(m)$ and those on boson lines have dimension mass squared $\left(m^{2}\right)$. 


\section{Acknowledgments}

The first author (MG) is partially supported by the Polish State Committee for Scientific Research, and by the Foundation for Polish Science. He would like also to express his gratitude to prof. A. Fäßler for his warm hospitality in Tübingen during the Summer 2006, and to prof. F. Šimkovic for help in correcting the manuscript.

\section{References}

1. H. L. Haber, G. L. Kane, Phys. Rep. 17 (1985) 75.

2. C. Aulakh and R. Mohapatra, Phys. Lett. B119 (1983) 136; G. G. Ross and J. V. Valle, Phys. Lett. B151 (1985) 375; J. Ellis et al., Phys. Lett. B150 (1985) 142; A. Santamaria, J. W. F. Valle, Phys. Rev. D39 (1989) 1780; A. Masiero, J. W. F. Valle, Phys. Lett. B251 (1990) 273.

3. M. A. Diaz, J. C. Romao, J. W. F. Valle, Nucl. Phys. B524, 23 (1998); A. Akeroyd et al., Nucl. Phys. B529, 3 (1998); A. S. Joshipura, M. Nowakowski, Phys. Rev. D51 (1995) 2421, ibid. 5271; M. Nowakowski, A. Pilaftsis, Nucl. Phys. B461 (1996) 19.

4. L. J. Hall, M. Suzuki, Nucl. Phys. B231 (1984) 419; R. Barbieri, D. E. Brahm, L. J. Hall, S. D. Hsu, Phys. Lett. B238 (1990) 86; H. Dreiner, G. G. Ross, Nucl. Phys. B410 (1993) 188; G. Bhattacharyya, D. Choudhury, K. Sridhar, Phys. Lett. B355 (1995) 193; G. Bhattacharyya, A. Raychaudhuri Phys. Lett. B374 (1996) 93; A. Y. Smirnov, F. Vissani Phys. Lett. B380 (1996) 317.

5. J. D. Vergados, private communication.

6. C. Liu, Mod. Phys. Lett. A 12 (1997) 329; R. Barbieri, M. M. Guzzo, A. Masiero, D. Tommasini, Phys. Lett. B 252 (1990) 251; O. Haug, J. D. Vergados, A. Faessler, S. Kovalenko, Nucl. Phys. B 565 (2000) 38; G. Bhattacharyya, H. V. KlapdorKleingrothaus, H. Päs, Phys. Lett. B 463 (1999) 77; A. Abada, M. Losada, Phys. Lett. B 492 (2000) 310; Nucl. Phys. B 585 (2000) 45; M. Góźdź, W. A. Kamiński, F. Šimkovic, Phys. Rev. D 70 (20004) 095005; M. Góźdź, W. A. Kamiński, F. Šimkovic, Int. J. Mod. Phys. E 15 (2006) 441; M. Góźdź, W. A. Kamiński, F. Šimkovic, Acta Phys. Pol. B 37 (2006) 2203; M. Góźdź, W. A. Kamiński, F. Šimkovic, A. Faessler, Phys. Rev. D 74, (2006) 055007. 